\title{
FACTORES QUE INCIDEN EN LA PARTICIPACIÓN SOCIAL DE LOS ADULTOS MAYORES EN ACTIVIDADES FÍSICO-RECREATIVAS
}

\author{
FACTORS AFFECTING SOCIAL PARTICIPATION OF OLDER ADULTS IN RECREATIONAL \\ PHYSICAL ACTIVITY
}

\section{Paulina Alegría V. ${ }^{1}$, Natalia Castillo N. ${ }^{2}$, Paulina del Solar D. ${ }^{3}$, Joaquín Varas R. ${ }^{4}$, Silvia Gómez L. , Pamela Gutiérrez M. ${ }^{6}$}

\section{Resumen}

Esta investigación se enmarcó en la temática de Ocupación y Adulto Mayor Activo, con el propósito de dar respuesta a la pregunta de investigación ¿Qué factores inciden en la participación social del Adulto Mayor en Actividades Físico-Recreativas?, orientándose a aquellos factores facilitadores $u$ obstaculizadores de dicha participación.

Para este objetivo realizamos entrevistas en profundidad a cuatro personas que cumplieron los criterios muestrales establecidos para el estudio, que corresponden a: Adultos Mayores que se reconozcan a sí mismos como activos, que participen hace al menos un año en Actividades Físico-Recreativas, como mínimo una vez por semana en el último año.

Para el análisis de datos de esta investigación cualitativa utilizamos la técnica de análisis de contenido desde el relato de los participantes, obteniendo como principales factores facilitadores de esta participación el intercambio de recursos no tangibles, la motivación, el cuerpo vivido, la trayectoria de vida, la habituación y la accesibilidad, como factores que influyen en la participación de adultos mayores.

Además de los mencionados existe una variedad de factores que interactúan entre sí, determinando atrayentes relaciones inter e intra factoriales, donde identificamos aspectos transversales a las distintas temáticas abordadas en esta investigación que posibilitan la apertura de nuevas líneas de investigación para la Ciencia de la Ocupación y Terapia Ocupacional.

\section{Palabras clave:}

Adulto Mayor - Actividad Físico Recreativa - Envejecimiento Activo - Participación Social - Calidad de vida - Factores, Ocupación

\section{Abstract}

This research was part of the issue of Occupation and Active Older Adult, setting a goal to answer the research question: What factors influence the social participation of the elderly, specifically in recreational physical activity?, aiming specifically those factors that emerge as facilitators or impede participation.

To achieve this objective, in-depth interviews were conducted to four people who met the criteria established for the study sample, corresponding to: Older adult who recognize themselves as active, involved at least a year in recreational physical activity, and as at least once a week in the last year.

This exploratory qualitative used the technique of content analysis from the account of participants, having as main findings of this participation facilitating factors, where the most significant relate to: Exchange of intangible resources, Motivation, Lived body, Path of

\footnotetext{
${ }^{1}$ Terapeuta Ocupacional, Lic. en Ciencias de la Ocupación. Universidad de Chile.paulina.alegría@gmail.com

${ }^{2}$ Terapeuta Ocupacional, Lic. en Ciencias de la Ocupación. Universidad de Chile. nataliacastillon@gmail.com

${ }^{3}$ Terapeuta Ocupacional, Lic. en Ciencias de la Ocupación. Universidad de Chile.paulina.dls@gmail.com

${ }^{4}$ Terapeuta Ocupacional, Lic. en Ciencias de la Ocupación. Universidad de Chile. j.varas.reyes@gmail.com

${ }^{5}$ Terapeuta Ocupacional, Lic. en Ciencias de la Ocupación, Académico Universidad de Chile. sgomez@med.uchile.cl

${ }^{6}$ Terapeuta Ocupacional, Lic. en Ciencias de la Ocupación, Universidad de Chile. Doctora en Psicología Social Universidad Autónoma de Barcelona. Contacto: pamelagutierrezm@med.uchile.cl
} 
life, Habituation and Accessibility, which relate primarily to factors relating to that person, being a powerful accessibility elderly external prism that influences their involvement in such activities.

In addition there was a variety of factors that interact with each other attractive determining relationships within and between categories, which identified issues which cut across the different themes addressed in this research.

Finally, note that the nature of this study enables the opening of new lines of research that may contribute to the Occupation Science and Occupational Therapy.

\section{Keywords}

Older Adults - Recreational Physical Activity - Active Aging - Social Participation -Quality Life - Factors - Occupation.

\section{INTRODUCCIÓN}

Según la Organización Mundial de la Salud (OMS), entre el año 2000 y 2050, la población mundial de 60 años o más se multiplicará más de tres veces, pasando de 600 millones a 2.000 millones. La mayor parte de ese aumento se producirá en países en desarrollo, donde pasarán de 400 a 1.700 millones de personas Adultas Mayores (OMS, s.f.). A nivel nacional, Chile se caracteriza por un progresivo aumento de la población Adulta Mayor, la que en el Censo del 2002 correspondió al 11,4\% de la población total (INE, 2002).

Respecto de las recomendaciones para esta situación, la OMS, en el año 2002, publica el documento "Envejecimiento activo: un marco político", donde identifica la práctica de la actividad física como predictor de calidad de vida. Esta asociación entre actividad física y calidad de vida, es reconocida también por entidades nacionales (Servicio Nacional del Adulto Mayor, 2010; Instituto Nacional del Deporte, 2013), sin embargo no pesquisamos en la búsqueda bibliográfica estudios específicos respecto a los factores que inciden en que un Adulto Mayor se constituya en un sujeto activo.

Cabe señalar que este aumento en la expectativa de vida de los Adultos Mayores, se asocia a un alto índice de sedentarismo, y de patologías crónicas asociadas (OMS, 2002, p. 84), lo que no implica una relación directa entre expectativa de vida y la calidad de vida.

Dada la relevancia de esta temática, en esta investigación profundizamos en torno al concepto de envejecimiento activo, orientándolo a la participación en Actividades Físico-Recreativas, entendidas éstas como "una ocupación voluntaria, un descanso de las demás actividades, e implica diversión, formación, participación social, desarrollo de la capacidad creadora y recuperación psicofísica". (Cagigal, 1981, citado en Buñuel, 1994, p. 99).

Como ya se ha señalado, y en relación a lo que el documento "Chile y sus Mayores: Resultados Segunda Encuesta Nacional de Calidad de Vida en la Vejez" (2010) refiere, la población adulta mayor en la actualidad se caracteriza por un nivel de sedentarismo que influencia negativamente su estado de salud y calidad de vida que escapa a la concepción de Adulto Mayor activo propuesto por la OMS. En relación a esto, planteamos como pregunta de investigación: ¿qué factores inciden en la participación social del Adulto Mayor en Actividades Físico-Recreativas? En respuesta a esta interrogante el objetivo general de la investigación es "Conocer dichos factores que inciden en la participación social del Adulto Mayor en Actividades Físico-Recreativas", identificando los factores facilitadores y obstaculizadores de esta participación.

Cabe destacar, que desde la Gerontología Social, Fernández Ballesteros (2009) clasifica las teorías del envejecimiento, a modo general, en biológicas ${ }^{7}$, sociológicas ${ }^{8}$ y psicológicas. De éstas, las teorías psicológi-

\footnotetext{
A pesar de que se valora su influencia en el proceso de envejecimiento, ya que éste se considera como universal, continuo, intrínseco, irreversible, que afecta a todos los seres vivos, y con características definidas por especie (Hoyl, M., s.f.:11), no presentan mayor vinculación con los hallazgos de la investigación, ya que aluden a procesos objetivos que no responden al relato de los Adultos Mayores participantes.

8 Dentro de las teorías sociales del envejecimiento destacan las referidas a la desvinculación (Cummings, 1961, citado en Fernández- Ballestero, 2009, p. 49), a la subcultura, y a la modernización (Fernández, 2009: p. 49), las que aportan a la
} 
cas compuestas por la teoría de la actividad y la continuidad, adquieren mayor relevancia para este estudio, y son abordadas en el apartado de discusión, ya que como la autora plantea, éstas permiten describir o predecir los cambios que con el tiempo y edad se producen en el funcionamiento psicológico de las personas, desde una perspectiva más subjetiva.

Por consiguiente, a raíz de la revisión del estado del arte surge evidencia en torno a los beneficios de la práctica de actividad física y la calidad de vida en el Adulto Mayor (OMS, 2002), pero no así de los factores que inciden en su involucramiento en estas actividades. Por lo tanto, planteamos que el conocer factores facilitadores y obstaculizadores, que inciden en la participación social del Adulto Mayor en este tipo de actividades, facilitará la generación de medidas para que este grupo poblacional tenga mayor acceso a dichas ocupaciones.

\section{Metodología}

La investigación se desarrolla desde el paradigma construccionista, con un enfoque cualitativo, que permite generar espacios de construcción de conocimientos de manera dialéctica, a través de lo que Rodríguez, Lorenzo y Herrera (2005) señalan como un proceso circular, que admite que en el transcurso de la investigación existan modificaciones, cambios, redefiniciones, aportes, y/o complementos que permiten enriquecerla.

Esta investigación se remite a la población Adulta Mayor, que incluye a toda persona de 60 años y más ${ }^{9}$, de la cual obtuvimos una muestra intencionada de cuatro personas de acuerdo a los siguientes criterios: Adultos Mayores que se reconozcan a sí mismos como $\operatorname{activos}^{10}$, que participen hace al menos un año en Actividades Físico-Recreativas, como mínimo una vez por semana en el último año.

Para acceder a la muestra realizamos primero una inmersión en el campo asistiendo al Campeonato Sud-

comprensión del proceso de envejecimiento, y son relevantes para el mismo, pero que no adquieren relevancia para esta investigación.

9 Criterio establecido por Naciones Unidas y asumido por el Servicio Nacional del Adulto Mayor, Gobierno de Chile.

10 Información obtenida desde el proceso de inmersión en el campo realizado por los investigadores, que es especificado en los párrafos siguientes. americano de Atletismo Máster y a la presentación de sedes de Adultos Mayores de La Florida, como un proceso previo a la selección de los Adultos Mayores participantes de este estudio, quienes recibieron y firmaron el consentimiento informado para el desarrollo de la investigación utilizando los datos aportados por ellos.

Cabe destacar, que el proceso de investigación lo desarrollamos en la Región Metropolitana de Santiago de Chile, entre los meses de octubre del año 2010 y diciembre del año 2011. La muestra contó con tres participantes de género masculino de nivel socioeconómico medio, de nombres ficticios Marcelo, quien es viudo y practica tenis; Carlos, quien es casado y practica pilates; y Tomás quien es separado y practica atletismo; y una persona de género femenino del mismo nivel socioeconómico, Marta, cuyo estado civil es viuda y practica gimnasia entretenida.

El proceso y estrategia metodológica lo llevamos a cabo en diferentes etapas, que incluyen desde lo propuesto por Valles (2007), un primer acercamiento al campo, registro etnográfico a través de notas de campo, y la confección y ejecución de una entrevista en profundidad estandarizada no programada. ${ }^{11}$

Finalmente, el proceso de producción de la información lo realizamos mediante la técnica de análisis de contenido (Rodríguez y cols., 2005), la cual se estructura en tres etapas cíclicas que comprenden la reducción de datos, disposición y transformación de los datos, obtención de resultados y verificación de conclusiones.

Basándonos en esto, confeccionamos un documento de análisis a partir de las notas de campo y las entrevistas en profundidad realizadas a los Adultos Mayores de la muestra, identificándolos con nombres ficticios, para resguardar los principios éticos que considera esta investigación. A partir de éste se generan documentos de vaciado, que incluyen en un primer momento todas las citas categorizadas, y en un segundo momento, las más representativas de cada categoría ${ }^{12}$. Cabe destacar que para fines metodológicos y analíticos agrupamos cada categoría con subcategorías, sin embargo para la

11 Que implica la realización de ensayos, estructuración de una pauta tipo, con preguntas de diferente índole, entrenar al equipo de investigadores y realizar una corrección técnica del método empleado. Cabe señalar, que se refiere a no programada ya que se usa un guión de entrevista dejando sitio para la libre expresión de ideas de los informantes, sin la necesidad de responder a las preguntas de manera rígida.

12 Documentos disponibles a través del mail seminariodetítuloam@ gmail.com. 
exposición de resultados y la discusión establecida en este artículo las consideramos de manera global.

\section{Resultados}

Desde el análisis cualitativo del relato de cada Adulto Mayor informante, generamos un proceso cíclico, caracterizado por el levantamiento de categorías, y por la revisión, redefinición, y reubicación constante de éstas.

Del proceso de categorización establecimos 11 categorías que corresponden a: Condición de salud, Características de la Actividad Físico-Recreativa, Habituación, Motivación, Ambiente físico, Trayectoria de vida, Accesibilidad, Intercambio de recursos no tangibles, Percepción personal, Características personales y Cuerpo vivido, las cuales en adelante se referirán como factores, puesto que las mismas se constituyen en facilitadores u obstaculizadores que buscan dar respuesta a los objetivos planteados.

De estos factores, clasificamos los hallazgos a la luz de los objetivos de este estudio, entre facilitadores y obstaculizadores de la participación, entregando en este apartado los resultados más significativos, entendiéndolos como los más incidentes y transversales desde el relato de los cuatro informantes.

Del proceso obtuvimos los factores de Intercambio de recursos no tangibles, Motivación, Cuerpo vivido, Trayectoria de vida, Habituación y Accesibilidad como los facilitadores que tienen mayor influencia en la participación social de los Adultos Mayores en Actividades Físico-Recreativas.

Además, desde el relato de cada informante en base a su experiencia personal, no se explicitan factores que impidan su participación. Sin embargo, al realizar el proceso de análisis consideramos que sí hay elementos que la ponen en riesgo, constituyéndose en factores obstaculizadores de la misma, y que serán profundizados posteriormente.

Corresponde además señalar que existe una relación constante intra e inter factores entre todos los aspectos categorizados.

Por consiguiente, para favorecer la comprensión de los hallazgos dividimos la presentación de resultados en tres partes: en primer lugar exponemos los factores facilitadores, en segundo lugar los obstaculizadores, y para finalizar, las interrelaciones de los mismos.

\section{Factores facilitadores}

Para presentar los resultados, expondremos una cita por cada categoría, luego definimos la misma desde la vinculación que se genera entre el reporte de los informantes, la teoría, y una síntesis de nuestro proceso reflexivo interpretativo, junto con los principales lineamientos del proceso de análisis y discusión.

\subsection{Intercambio de recursos no tangibles:}

$$
\begin{aligned}
& \text { "... se abren muchos mundos, conoces } \\
& \text { muchas personas, muchas actividades, } \\
& \text { ves otras formas de ver la vida, ves } \\
& \text { que existen otros intereses y otras } \\
& \text { motivaciones y vas descubriendo mil } \\
& \text { cosas..." (Marcelo, cita } 3: 37) .{ }^{13}
\end{aligned}
$$

Entendemos esta categoría como la relación de reciprocidad que se establece entre el Adulto Mayor que participa de Actividades Físico-Recreativas y los distintos grupos sociales con que se vincula: familiares, pares y compañeros, e integrantes de la comunidad, la cual se caracteriza por un flujo constante de aspectos subjetivos no materiales de manera recíproca.

En consecuencia, de acuerdo a lo anteriormente expuesto, la interacción con compañeros se vislumbra como el factor facilitador más significativo, refiriéndose a la incidencia de la participación social de los Adultos Mayores en Actividades Físico-Recreativas, pues el espacio de relación que se genera en el marco de estas actividades permite que emerjan redes de apoyo como parte de un círculo virtuoso entre la participación de los Adultos Mayores, su interacción con compañeros y los fenómenos asociados a las mismas.

\subsection{Motivación}

"... es que mañana tengo que hacer tal cosa, y cuando pueda, es que no, hace mucho frío, hace mucho calor, tengo que comprar, siempre se pone algo, pero es

\footnotetext{
13 Cita perteneciente al documento de "Vaciado por categoría" utilizado en el proceso de análisis de datos cualitativos. Éste se genera a partir del "Documento de análisis" base para la investigación, que se encuentran disponibles a través del mail seminariodetítuloam@gmail.com
} 
una inercia en que uno el momento que la rompe ya se acabó, es el empujón..." (Marcelo, cita 1:37).

Desde el análisis realizado, entendemos por motivación el deseo del Adulto Mayor por la incursión, inmersión y continuidad del mismo en Actividades Físico-Recreativas.

De esta forma, la motivación es un factor facilitador que incide en la participación social de los Adultos Mayores en Actividades Físico-Recreativas. Cabe destacar que existen diferencias cualitativas respecto a la motivación, observándose que por una parte, la motivación extrínseca está más asociada al involucramiento inicial en estas actividades, y que la motivación intrínseca además de relacionarse con este primer acercamiento, juega un papel fundamental en la mantención en dichas actividades.

\subsection{Cuerpo vivido}

"...Las inquietudes de niño eran el sentirme libre, el correr contra el viento o el andar en bicicleta contra el viento es una sensación demasiado especial..." (Marcelo, cita 3: 33).

Entenderemos por cuerpo vivido como la experiencia de cada Adulto Mayor de ser y experienciar la Actividad Físico-Recreativa a través del propio cuerpo.

Como se infiere de la lectura de esta cita, el cuerpo vivido constituye un factor facilitador para la participación social y mantención de los Adultos Mayores en Actividades Físico-Recreativas, ya que se vislumbra desde el relato de los informantes que el experimentar sensaciones satisfactorias al desempeñar estas actividades, y genera mayor motivación para involucrarse en las mismas.

Desde los relatos observamos que la experiencia del cuerpo vivido inevitablemente se relaciona y se enriquece mediante la interrelación con otros factores, fenómenos que expondremos posteriormente.

\subsection{Trayectoria de vida}

"...Facilita que esté jubilado y tengo tiempo. De vez en cuando falto, por cosas muy puntuales, pero el estar jubilado te permite estar más libre, hago muchas cosas que no hice cuando trabajaba, ahora las hago porque me alcanza bien el tiempo..." (Carlos, cita 6: 52)

"... a mí me sirve mucho porque yo soy sola, tengo tres hijos y uno vive conmigo no más, soy viuda, entonces mi vida la ocupo, me ocupo en hacer algo útil..." (Marta, cita 3:24).

A raíz del análisis realizado, entendemos trayectoria de vida como los eventos, sucesos, y características vinculadas a las distintas etapas del ciclo vital que ha cursado el Adulto Mayor, y su influencia en la participación en Actividades Físico-Recreativas.

Este factor comprende tanto los eventos asociados a la etapa de adultez mayor, como los ligados a la historicidad y las expectativas de los informantes, los cuales constituyen factores facilitadores de la participación social de los Adultos Mayores en Actividades Físico-Recreativas. No obstante lo anterior, desde el análisis emerge que existen diferencias ligadas al género y a componentes generacionales que determinan el primer acercamiento a estas actividades, destacando que en los participantes de género masculino, la trayectoria de vida se asocia a roles productivos, iniciando su participación de manera temprana, en espacios particulares pagados; y que por otra parte la participante de género femenino se incorpore de manera más tardía al cesar sus roles de crianza, y de cónyuge, en instancias municipales y gratuitas, como se refleja en las citas expuestas previamente.

\subsection{Habituación}

"...Sí, yo me encuentro activa, eso es
cierto. No estoy más de una hora en la
casa, menos sentada. Yo era muy buena
para tejer antes, pero ahora ya no quiero
saber nada de tejido, porque es sentarse
ahí y listo. De que sirve... sirve... pero sí
hay cosas que a uno le pueden ocupar la
mente en otra cosa... para mí tejiendo la
mente trabaja menos..." (Marta, cita 1:31).

La habituación la definimos como la adquisición y configuración de hábitos y roles en el desempeño ocupacional cotidiano de los Adultos Mayores, considerando su participación en Actividades Físico-Recreativas.

A partir de la cita planteada, es posible inferir la trascendental relación de los hábitos y roles de cada perso- 
na con la estructuración de rutina, e incorporación de estas actividades en su repertorio ocupacional, en una suerte de proceso circular. Además, este factor se liga al género, la trayectoria de vida, y la búsqueda de nuevas redes de apoyo del grupo informante.

\subsection{Accesibilidad}

\begin{abstract}
"...en otros casos el estado o las municipalidades deben fomentar y poner recintos a disposición de la gente, o sea recintos y profesores..." (Tomás, cita 2:12).
\end{abstract}

Entendemos la accesibilidad como los medios disponibles vinculados al Adulto Mayor, que incluyen las oportunidades de participación, programas, instancias de difusión, recursos en la comunidad y recursos económicos personales, que le otorgan la posibilidad de participar en Actividades Físico-Recreativas.

Como se refleja en esta cita, la accesibilidad es un factor que influye en la participación de los Adultos Mayores en Actividades Físico-Recreativas, tornándose como un facilitador distinto según el género y rol de cada Adulto Mayor. Para los de género masculino los recursos económicos personales se constituyen en el principal medio para acceder a estas actividades, mientras que para la participante de género femenino los recursos comunitarios para la participación favorecen su involucramiento de manera gratuita. Por otra parte, los programas, la difusión y las oportunidades de participación, en suma, se constituyen en un factor que favorece la participación.

\section{Factores obstaculizadores}

Como mencionamos anteriormente, estos factores no son referidos desde los entrevistados como una barrera para el involucramiento en Actividades Físico-Recreativas. Aun así, reconocemos desde el relato de los Adultos Mayores y análisis efectuado, que sí generan tensión en la participación de los mismos.

De esta forma, la trayectoria de vida emerge como un factor obstaculizador temporal para la persona de género femenino, así como sus recursos económicos, en términos de que determinan un involucramiento más tardío en este tipo de actividades, según cese de roles de manejo y cuidado del hogar, de crianza, y de pareja. Ello puede vincularse al empobrecimiento de las mujeres, que ven acotada su participación a instancias gratuitas o en su defecto de bajo costo.

A su vez, el apoyo familiar se constituye en un factor influyente para el participante que se encuentra casado, ya que como se vislumbra en la cita "...el único pero que me dice es que la dejo sola, pero yo igual voy no más..." (Carlos, cita 1, página 54), su pareja le ofrece cierto grado de resistencia, pero este aspecto no llega a tomar fuerza por la potencia que tienen los facilitadores para su participación.

Cabe destacar que los informantes son capaces de visualizar factores obstaculizadores en terceros, que no participan en Actividades Físico-Recreativas, refiriendo el ambiente físico en términos de condiciones ambientales, la motivación, o la preferencia de ocupaciones más sedentarias.

\section{Interrelaciones}

A continuación, daremos a conocer las principales interrelaciones y conclusiones de las categorías previamente planteadas, pues éstas emergen de manera paralela y poseen un alto nivel de relevancia para la investigación, ya que permiten tener una comprensión más acabada, e integral del objeto de estudio y los datos presentados previamente.

La interrelación entre los factores que inciden en la participación social de los Adultos Mayores en Actividades Físico-Recreativas, se encuentra influenciada de manera transversal por las variables generacional y de género así como por la cultura en que los entrevistados se formaron. Éstos al involucrarse en dichas actividades generan un desempeño ocupacional ${ }^{14}$ (DO) particular, que se configura de forma más significativa en torno a las seis categorías previamente abordadas. Cabe destacar que esta relación es bidireccional, y que no excluye la relación circular existente entre todas las categorías enunciadas (ver figura 1).

Además, consideramos que estas variables transversales determinan el distinto sentido y significado que cada Adulto Mayor otorga a la Actividad Físico-Recrea-

\footnotetext{
14 "El desempeño ocupacional es el resultado de la interacción de la persona, el ambiente y la ocupación es una experiencia dinámica y siempre cambiante de una persona que participa en actividades, tareas y ocupaciones con propósito dentro de un ambiente". (Stewart, D.; Letts, L.; Law, M.; Acheson, B.; Strong, S.; Rigby, C. (2005) en Crepeau, E.; Cohn, E.; Schell, B.).
} 
tiva en un momento determinado de su vida, y por tanto inciden en su incorporación y permanencia en éstas. Lo anterior, permite destacar la influencia entre la historicidad y experiencia en Actividades Físico-Recreativas, y el dinamismo propio de cada factor, que genera significados y expectativas que varían en el tiempo según la vivencia personal y subjetiva de cada persona.

De hecho, los eventos normativos propios de la etapa del ciclo vital que cursa la muestra, determinan que la participante de género femenino al cesar los roles de crianza, y al aumentar el tiempo para sí misma, busque instancias de participación social asociadas a su necesidad de interactuar con terceros, emergiendo las Actividades Físico-Recreativas como una opción potente, que fomenta una configuración de redes de apoyo e incentiva el sentido de pertenencia, que contribuyen a su motivación intrínseca para mantenerse vinculada a la actividad. Todo esto se remite al plano de los recursos comunitarios, ya que la carencia de recursos económicos personales, ligada a los roles desempeñados en su trayectoria de vida, determinan su oportunidad de acceso a instancias de participación gratuita en el plano local en esta etapa de su vida.

Finalmente el cuerpo vivido de la misma se experimenta en tanto el sí mismo se relaciona con los demás en el espacio de interacción que se genera dentro de la actividad en que se involucra.

Por su parte, el género masculino posee una incorporación más temprana a este tipo de actividades a través de recursos económicos personales ligados a los roles productivos que desempeña durante su vida. A su vez, las motivaciones que lo movilizan a involucrarse y permanecer en las actividades son predominantemente intrínsecas, y presentan relación con la vivencia individual de los sujetos en la realización de la actividad, y su ser a través del cuerpo en las mismas.

Figura $N^{\circ} 1$ : Relaciones entre los factores facilitadores.

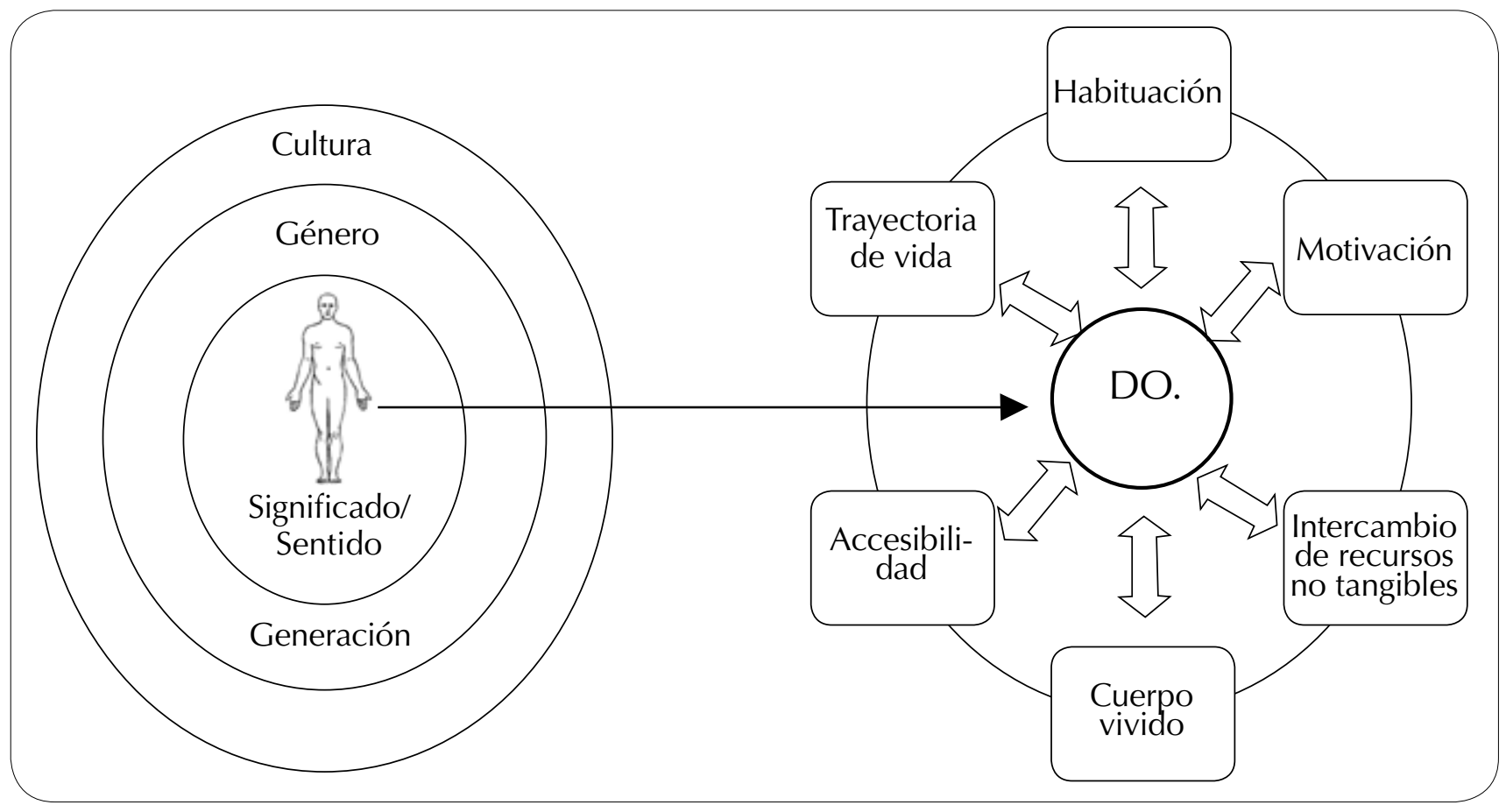

Descripción: Interrelaciones de los factores que inciden en la participación social de los Adultos Mayores en Actividades Físico Recreativas, que emergen del análisis de datos cualitativos.

\section{DisCUSIÓN Y CONCLUSIONES}

Como mencionamos previamente, además de los factores descritos como facilitadores y obstaculizadores, 
surgen variables transversales que influencian de manera particular en cada Adulto Mayor participante. En este marco, encontramos variables como el género y la generacionalidad, configurados sobre una cultura particular, aspectos que se condicen con lo que plantea la OMS (2002), donde destaca la cultura y el género como determinantes transversales del envejecimiento activo.

A continuación, abordaremos estas variables contrastándolas con el relato, la literatura y nuestra interpretación.

Vinculando la variable de género ${ }^{15}$ y el factor de habituación, encontramos que la informante de género femenino mantiene una tendencia a desempeñar roles simultáneos en la etapa del ciclo vital que cursa actualmente, haciendo la salvedad que al vivir eventos normativos como el "nido vacío", o la "viudez", aumenta su repertorio ocupacional incluyendo tanto ocupaciones previas de manejo y mantención del hogar, como actividades recreacionales que responden a las motivaciones de mantenerse vinculada a distintos grupos sociales, y al mayor nivel de autonomía que vivencia. Todo esto se sustenta con lo que señala el programa de las Naciones Unidas para el desarrollo respecto a que ".. No habría una conformación del tiempo libre independiente y autónomo de las actividades y responsabilidades correspondientes a otros ámbitos. Las mujeres experimentan el tiempo libre como una yuxtaposición o simultaneidad entre varios tiempos". (PNUD, 2010, p: 79) ${ }^{16}$.

Ahora bien, vinculando el factor de trayectoria de vida y la historicidad, con el género masculino, hemos mencionado que en la muestra existe una participación constante en la Actividad Físico-Recreativa, y que perciben un mejor estado de salud y calidad de vida, destacando en el relato de los Adultos Mayores informantes los beneficios que de esta participación se extraen. Ade-

15 El PNUD define género como "los atributos sociales y las oportunidades asociadas con el ser femenino y masculino $y$ las relaciones entre mujeres y hombres, niñas y niños, como también entre las mujeres y entre los hombres. Estos atributos, oportunidades y relaciones están construidas socialmente, son aprendidas a través de procesos de socialización, y varían según el contexto social y temporal. El género determina lo que puede esperarse, lo que es permitido y valorado en una mujer o un hombre en un contexto dado" (PNUD 2001b).

16 Informe anual del Programa de las Naciones Unidas para el Desarrollo 2010, "Desarrollo humano en Chile. Género: Los desafíos de la Igualdad". Este informe presenta aspectos relativos a la población general, pero que se contrastan con los hallazgos de esta investigación cuando se contrasta la variable de género y las influencias que de ésta se deslindan. más, éstos reconocen en el género femenino una falta de información respecto a los beneficios de estas actividades, y por ende aseveran que ellas se involucren en menor medida. Lo anterior se respalda con los aspectos señalados en la Encuesta Nacional de Calidad de Vida en la Vejez, que plantea que "los hombres mayores y quienes tienen mayores niveles educacionales realizan más ejercicio físico". (Pontificia Universidad Católica de Chile, SENAMA-Caja Los Andes, 2010, p. 72), y que los hombres con mayor nivel educacional son los que tienen mejor percepción sobre su salud." (Pontificia Universidad Católica de Chile \& cols. 2010, p. 52).

Al referirse al intercambio de recursos no tangibles, se señala de manera transversal que la interacción con compañeros se presenta como un factor facilitador, pues desde los resultados se torna significativa, para involucrarse y mantenerse participando, rescatando la sociabilidad dentro de este espacio, ya que "una mayor sociabilidad puede favorecer una mayor calidad del tiempo libre en la medida en que la participación en organizaciones sociales -como clubes deportivos o juntas de vecinos-y compartir con los amigos propician que el tiempo sea un momento de disfrute, fuera de las preocupaciones del hogar y el trabajo. Y, a la inversa, el tiempo libre de calidad crea oportunidades concretas para desarrollar la sociabilidad". (PNUD, 2010, p. 80). Por tanto, una mayor sociabilidad, según lo citado, favorece la motivación del Adulto Mayor por vincularse a un grupo de pertenencia, y con ello aumentar las redes de apoyo que en esta etapa tienden a verse disminuidas.

Paralelamente, al referirnos a la motivación intrínseca como un factor incidente en la participación social de los Adultos Mayores en Actividades Físico-Recreativas, consideramos que las características de los programas en que los informantes se involucran, favorecen su participación, ya que son propuestas flexibles, que permiten a los Adultos Mayores hacerse parte de ellas independiente de posibles limitaciones y/o eventualidades que puedan tener, alcanzando un estado de flujo, según lo propuesto por Csikszentmihalyi en la Teoría de Flujo (1975, citado en Barberá, 2002), que “...incorpora la noción de flujo, como punto de encuentro entre el nivel de dificultad de la tarea, característico de una actividad, y las habilidades de las que dispone la persona para resolver la situación problemática. Aquí, la activación motivacional no depende sólo de la novedad o interés intrínseco del trabajo en cuestión, sino de la correspondencia entre ésta y los recursos personales de los que se dispone para afrontar la situación...". Por lo tanto, el nivel de dificultad de la Actividad Físico- 
Recreativa en que participa cada Adulto Mayor será personal, lo que favorece su motivación intrínseca y por ende, la mantención en este tipo de actividades.

Si bien lo analizado hasta ahora se ha enfocado en publicaciones que respaldan nuestros hallazgos, surge otra línea analítica, en que emergen relaciones con aspectos psicológicos vinculados a la participación social de los Adultos Mayores en Actividades Físico-Recreativas, los que se refieren a la trayectoria de vida de los informantes, que serán abordados a continuación.

Al referirnos a la definición de Actividad Físico-Recreativa utilizada en la investigación, destacamos que ésta se constituye respecto de lo que la persona concibe con la participación, no de la naturaleza de la actividad misma. Así observamos que los entrevistados participan de distintas Actividades Físico-Recreativas como tenis, atletismo, pilates y gimnasia entretenida, variando las percepciones personales y significados que para cada uno poseen, los que no dejan de ser relevantes, pues determinan su involucramiento y mantención en estas ocupaciones. De esta forma, consideramos que existe una relación con la teoría psicológica del envejecimiento referida a la actividad (Lemon 1972, citado en Fernández, 2009, p. 48), en la cual se propone que para el sujeto lo más importante son las actividades que tienen sentido para él y no la actividad por sí misma, por tanto, no es importante la cantidad de interacciones que mantenga sino su significación.

A su vez, la Teoría de la Continuidad (Madox, 1963; Atchley 1989, 1999, citado en Fernández-Ballesteros, 2009, p. 48), asociada a la anterior, relaciona pasado, presente y futuro y los patrones que pueden ser anticipados sobre formas de pensar, actuar y relacionarse de los Adultos Mayores, de tal forma que el paso a la vejez es una prolongación de experiencias, proyectos y hábitos de la vida. Todo esto se relaciona con la categoría de Trayectoria de vida, y Habituación, pues a partir del relato de los participantes inferimos que los hábitos y roles pasados, y el significado de los mismos en sus vidas, determinan la continuidad y adaptación de estas personas a la etapa del ciclo vital que cursan, manteniendo y proyectando su participación en las Actividades Físico-Recreativas a lo largo de su vida.

Además, a partir de la vinculación de nuestros resultados con esta teoría, surge la reflexión de cómo las oportunidades de participación determinan la accesibilidad de los Adultos Mayores a estas actividades, destacando que los participantes de género masculino se incluyen en su desempeño ocupacional desde etapas más tempranas, y que en el género femenino, su in- corporación sea una vez iniciada esta etapa del ciclo vital. Cabe destacar que en los informantes masculinos esta participación genera hábitos asociados, pues son ocupaciones arraigadas a ellos y a su rutina diaria, por lo que mantienen un estilo de vida saludable en el transcurso de las mismas. En consecuencia, consideramos importante generar instancias de participación desde etapas previas a la adultez mayor, ya que según nuestros hallazgos las personas que se incorporan más tempranamente a estas actividades son las que poseen un desempeño ocupacional que se condice con lo que postula la teoría de la continuidad, y que perciben una mejor calidad de vida.

A modo de conclusión, destacamos factores que inciden en la participación social del Adulto Mayor en Actividades Físico-Recreativas, siendo facilitadores el intercambio de recursos no tangibles, motivación, cuerpo vivido, trayectoria de vida, habituación, y accesibilidad, y obstaculizadores el apoyo familiar, condiciones ambientales, y recursos económicos personales, los que se encuentran influidos por las variables de género, generacionales, y culturales que finalmente poseen incidencia en el significado que cada Adulto Mayor le otorga al involucrarse en estas actividades.

En vista de todos estos aspectos, consideramos que frente a la misma temática abordada mediante la pregunta de investigación, en otro grupo de Adultos Mayores sería posible identificar tantos factores y fenómenos, como diversidad de Adultos Mayores existe. Entendiendo esto, es que reconocemos que los hallazgos que surgen a raíz de esta investigación, representan una realidad acotada a características particulares de la muestra, en relación a múltiples variables socioculturales, sociohistóricas y personales.

Lo anterior,, resalta la variabilidad propia del ser humano, y por ende intenta abrir nuevos caminos de construcción de práctica, teoría y práctica, una vez conocido y re- conocido el campo de estudio, recalcando en este punto la riqueza de la investigación cualitativa, en términos de que nos permite conocer a fondo la diversidad de factores que influyen en la conducta humana desde la realidad propia de cada persona, sin reducirla a aspectos cuantitativos, que se consideran necesarios, pero una vez valorada la experiencia personal y subjetiva de los participantes de la investigación.

Finalmente, como en todo proceso de aprendizaje es válido mirarse a sí mismo y reconocer aquellos aspectos que quedan al margen de esta investigación y pueden ponerse en cuestión. Desde el punto de vista metodológico reconocemos que en la selección de la 
muestra podrían haberse considerado aspectos como el nivel socioeconómico, o igualdad en el número de informantes por género, sin embargo, fueron aspectos que emergieron del campo, y que podrían ser abordados en investigaciones posteriores. Además, consideramos que el análisis se habría enriquecido si hubiésemos ahondado en mayor medida en los factores obstaculizadores de la participación de la muestra, no obstante se cumple con los objetivos de este estudio, dejando abiertas futuras líneas de investigación. Así mismo, la dicotomía de los objetivos planteados influyó en el proceso de análisis y levantamiento de categorías/factores, que nos llevaron a focalizarnos en los facilitadores de la participación de la muestra en estas actividades.

Reconocemos que tanto Terapia Ocupacional como Ciencia de la Ocupación aportan desde la atención primaria a la prevención y promoción de la salud, incorporando en ello la relación existente entre los factores mencionados en los resultados de este estudio, y los conceptos y acciones propios de nuestra disciplina, dentro de las cuales damos énfasis a la promoción de hábitos saludables, la orquestación de una rutina basada en ocupaciones significativas, y otras medidas tendientes a un estilo de vida activo, todo esto con el fin de fomentar una mejor calidad de vida de este grupo etario.

En consecuencia, emergen variados cuestionamientos que resultaría interesante abordar en estudios posteriores, indagando género, nivel socioeconómico, entre otros, ya que permitirían una mayor profundización del área, y favorecerían medidas tendientes a mejorar la calidad de vida de un grupo poblacional que, como se ha mencionado, se encuentra en aumento, con un alto nivel de sedentarismo y bajos niveles de participación en este tipo de actividades, y por tanto, cada vez más necesario de estudiar.

Si bien es cierto, el objetivo del presente estudio fue conocer los factores que inciden en la participación social del Adulto Mayor, específicamente en Actividades Físico-Recreativas, consideramos que es un aporte y punto inicial para futuros estudios relacionados a esta área, o más aún, para ser una variable a considerar en la toma de decisiones a nivel macro y gubernamental, con el fin de promover la adultez mayor activa y con ello la calidad de vida de este grupo etario.

\section{ReferenCiAs BibliográficAS}

Barberá, E. (2002). Modelos explicativos en psicología de la motivación. Revista Electrónica de Motivación y Emoción, Volumen 5, Número 10. Mayo 2002. Extraído de http://reme. uji.es/articulos/abarbe7630705102/texto.html en diciembre de 2011.

Buñuel, A. (1994). La construcción social del cuerpo de la mujer en el deporte, Revista española de investigaciones sociológicas, № 68, págs. 97-118.

España. Extraído de http://dialnet.unirioja.es/servlet articulo?codigo=768117 en Septiembre de 2011.

Fernández Ballesteros, R. (2009). En Gerontología Social (págs. 43-50). España: Pirámide.

Gutiérrez, P. y Vidaña, L. (2007). Capítulo 29. Desarrollo de marcos conceptuales de Terapia Ocupacional: Modelo de Ocupación Humana de Gary Kielhofner. Pág. 376-393. En el libro: Terapeutas Ocupacionales. Servicio Andaluz de Salud. Editorial MAD. España.

Hoyl, M. (s.f.). Envejecimiento Biológico, Capítulo 2: Proceso de envejecimiento: sus implicancias biológicas y sociales, Manual de Geriatría, página 11. Extraído de http://escuela. med.puc.cl/publ/ManualGeriatria/PDF/EnvejeBiologico.pdf el 23 de diciembre de 2010.

IND. Instituto Nacional de Deportes (s.f.). Deporte recreativo recuperado en marzo 31, 2013, a partir de http://www.ind.cl/ deporte-masivo/deporte-recreativo/Paginas/Inicio.aspx

Instituto Nacional de Estadísticas (2002). Cifras Mayores, Extraído de http://www.ine.cl/canales/chile_estadistico/estadisticas_ sociales_culturales/adultosmayores/pdf/cifrasmayores.pdf

Massone, F. (2010). Experiencias del cuerpo en movimiento durante la vejez: Actividad Física Recreativa y calidad de vida en adultos mayores de la comuna de Ñuñoa. Memoria para optar al Título de Antropóloga Social, Departamento de Antropología, Facultad de Ciencias Sociales, Universidad de Chile.

Montes de Oca, V. (2003). Redes de apoyo social de Personas Mayores: elementos teórico-conceptuales, ponencia dictada en el curso Calidad de Vida de Personas Mayores: instrumentos para el seguimiento de Políticas y Programas, extraído de http://www.eclac.cl/celade/noticias/paginas/7/13237/ PP18. pdf el 6 de diciembre de 2011.

OMS. (s.f.). OMS. ¿Qué repercusiones tiene el envejecimiento mundial en la salud pública? Recuperado noviembre 2, 2010, a partir de http://www.who.int/features/qa/42/es/index.html

Organización Mundial de la Salud (OMS). Envejecimiento Activo: Un marco político, página: 82- 84. Extraído desde http://ccp. ucr.ac.cr/bvp/pdf/vejez/oms_envejecimiento_activo.pdf en octubre de 2011. 
Papalia, D.; Wendkos, S.; Duskin R. (2010). Capítulo 18: Desarrollo Psicosocial de la Adultez Tardía, Desarrollo Humano, páginas 592-593. Edición Nº11, Editorial Mc GrawHill, México.

Parkinson, S.; Forsyth, K.; Kielhofner, G. (2004). Capítulo Uno: Bases Teóricas del MOHOST: El Modelo de Ocupación Humana, Manual del Usuario de Perfil Ocupacional del Modelo de Ocupación Humana (MOHOST) (Versión 2.0), página 7. Chicago, Universidad de Illinois.

Pontificia Universidad Católica de Chile, Servicio Nacional del Adulto Mayor, Caja Los Andes (2010). Chile y sus Mayores: Resultados Segunda Encuesta Nacional de Calidad de Vida en la Vejez. Extraído de http://www.senama.cl/filesapp/ChileYsusMayores_EncuestaCalidaddeVida.pdf en octubre 2011.

Programa de las Naciones Unidas para el Desarrollo (PNUD) Chile (2010). Capítulo 6: Las representaciones de género y su impacto en las prácticas cotidianas. Desarrollo Humano en Chile, Género: los desafíos de la igualdad, página 79-82. Santiago, Chile.

Rodríguez, C.; Lorenzo, O.; Herrera, L. (2005). Teoría y Práctica del Análisis de Datos Cualitativos. Proceso general y criterios de calidad, Revista Internacional de Ciencias Sociales y Humanidades, SOCIOTAM, julio-diciembre, año/vol. XV, número 002, Universidad Autónoma de Tamaulipas, México, pp. 133-154. Extraído de https://www.ucursos.cl/ medicina/2010/1/TOMETI24/1/material_docente/.

San Martín Petersen, C. (2007). Espiritualidad en la Tercera Edad, Psicodebate 8, Cultura y Sociedad, pág 111. Extraído de http://www.palermo.edu/cienciassociales/publicaciones/ pdf/Psico8/8Psico\%2008. pdf el 5 de diciembre de 2011.

Servicio Nacional del Adulto Mayor (s.f.). Glosario Gerontológico, página 2. Extraído de http://www.senama.cl/filesapp/GLOSARIO_GERONTOLOGICO.pdf el mes de octubre de 2010.

Servicio Nacional del Adulto Mayor (s.f.) Misión. Recuperado en octubre de 2010. A partir de http://www.senama.cl/NuestraMision.html

Stewart, D.; Letts, L.; Law, M.; Acheson, B.; Strong, S.; Rigby, P. Capítulo 18: Teorías derivadas de las perspectivas del comportamiento ocupacional. En Crepeau, E.; Cohn, E.; Schell, B. (2005). Willard y Spackman. Terapia Ocupacional, página 229. Buenos Aires. Médica Panamericana.

Valles, M. (2007). Técnicas cualitativas de investigación social, páginas 168-172, 184-189, 203-210). España: Síntesis. 